\title{
1 Normative data for peripapillary retinal nerve fibre layer OCT scans in patients of African descent.
}

2 Frederick R. Burgess ${ }^{1,2}$, Kazim Dhalla ${ }^{3}$, Celina F. Mhina ${ }^{4}$, Andrew Blaikie ${ }^{1}$

3

$4 \quad{ }^{1}$ Dept. of Global Health, School of Medicine, University of St Andrews, U.K.

$5 \quad{ }^{2}$ Dept. of Ophthalmology, Princess Alexandra Eye Pavilion, Edinburgh, U.K.

$6 \quad{ }^{3}$ Dr Agarwal's Eye Hospital, Dar es Salaam, Tanzania

$7 \quad{ }^{4}$ Muhimbili University of Health \& Allied Sciences, Dar es Salaam, Tanzania

8

9 Corresponding author:

10 Dr Frederick R. Burgess

11 University of St Andrews, School of Medicine, North Haugh, St Andrews, Fife, U.K.

12 frb2@st-andrews.ac.uk

$13+44(0) 7773319101$

14

15 We present normative OCT peripapillary data for healthy adults of African descent and wish to emphasise the lack of normative control data from all regions of sub-saharan Africa. 
18 We read with interest the recent article by Yashadhana et al calling for improved diversity in global eye health leadership (1). Leadership is not the only area of eye health where diversity could be improved. We are currently investigating Tanzanian Endemic Optic Neuropathy (TEON): a bilateral subacute optic neuropathy of unknown aetiology affecting young people in Tanzania (2). The condition demonstrates similar clinical and temporal characteristics to Leber's Hereditary Optic Neuropathy (LHON). As part of the TEON Study - a collaborative research project that aims to identify the causes of TEON - we sought to compare the optical coherence tomography (OCT) findings of TEON and LHON, utilizing a new OCT scanner (Topcon 3D OCT-1 Maestro, Topcon Medical Systems, Oakland, NJ, USA) at Muhimbili National Hospital.

To allow thorough comparison of OCT data we need a representative normative dataset. Only $20 \%$ of the normative dataset of our OCT analysis software contains data from people of African descent (AD) and only $45 \%$ were under the age of 40 (3). The African Descent and Glaucoma Evaluation Study (ADAGES), a study that investigated glaucoma in American AD patients, showed significant baseline differences in optic nerve parameters between healthy participants of African and European descent (4).

We therefore conducted a literature search for normative data for OCT peripapillary retinal nerve fibre layer ( $p R N F L$ ) thickness measurements in healthy adult AD participants, the results of which can be seen in Table 1.

Table 1. Studies reporting normative pRNFL OCT data for adult participants of African descent.

\begin{tabular}{|c|c|c|c|c|c|c|c|c|c|}
\hline Study first author & Location & Year & Eyes $(n=)$ & Weight* & pRNFL & tRNFL & iRNFL & sRNFL & nRNFL \\
\hline Mashige & South Africa & 2017 & 600 & 11.88 & 110 & 73.63 & 135.06 & 131.96 & 87.24 \\
\hline Girkin & USA & 2010 & 315 & 6.24 & 103.69 & 66.48 & 135.12 & 128.83 & 84.3 \\
\hline Sani & Nigeria & 2016 & 220 & 4.36 & 104.2 & 67.2 & 129.2 & 135.3 & 85.1 \\
\hline Ismail & South Africa & 2019 & 132 & 2.61 & 108.7 & 74.8 & & & 77.7 \\
\hline Blumberg & USA & 2016 & 103 & 2.04 & 93.98 & 60.59 & 122.71 & 119.16 & 73.42 \\
\hline Ocansey & Ghana & 2020 & 100 & 1.98 & 102.4 & 66.8 & 133.6 & 131.5 & 76.7 \\
\hline Knight & USA & 2012 & 51 & 1.01 & 93.9 & 57.8 & 125.2 & 119.6 & 73 \\
\hline Rao & USA & 2015 & 50 & 0.99 & 97.18 & & 130.04 & 121.16 & \\
\hline Racette & USA & 2005 & 42 & 0.83 & 114.86 & 84.55 & 141.69 & 149.79 & 82.86 \\
\hline Poon & USA & 2018 & 40 & 0.79 & 92.97 & 62.09 & 118.65 & 114.47 & 76.49 \\
\hline Seraji-Bozorgzad & USA & 2016 & 27 & 0.53 & 100.9 & 68.9 & 133.7 & 128.1 & 72.4 \\
\hline Budenz & USA & 2007 & 27 & 0.53 & 101.1 & & & & \\
\hline Alasil & USA & 2013 & 26 & 0.51 & 99.2 & & & & \\
\hline Kimbrough & USA & 2015 & 14 & 0.28 & 100 & 67 & 135 & 126 & 73 \\
\hline \multicolumn{3}{|c|}{ Weighted average (AD participants) } & & & 105.0 & 69.6 & 132.6 & 130.1 & 82.9 \\
\hline \multicolumn{5}{|c|}{ OCT software average (all ethnicities) } & 104.0 & 70.6 & 123.9 & 116.4 & 80.6 \\
\hline \multicolumn{10}{|c|}{ *Weight based on sample size relative to the median sample size. } \\
\hline & & & & & & & & & \\
\hline
\end{tabular}

The difference between the averages is in agreement with studies that have previously examined ethnic differences in PRNFL OCT thicknesses - namely that AD patients have thinner temporal peripapillary RNFL thicknesses and thicker RNFL in the other quadrants (5). 
It should be noted that many of these RNFL studies concern glaucoma, primarily a disease of the elderly, hence normative data may be skewed by age, as well as differences in scan equipment, scan technique, and optic disc morphology.

The variation in RNFL thicknesses seen in Table 1 shows the difficulties in comparing OCT scan data and emphasises the need for more extensive normative control data from all regions of sub-saharan Africa. This article hopefully highlights the heterogenous nature of the term 'African descent' and that there are no published normative datasets for East African patients.

\section{References}

1. Yashadhana A, Zhang $\mathrm{JH}$, Yasmin $\mathrm{S}$, Morjaria $\mathrm{P}$, Holland $\mathrm{P}$, Faal $\mathrm{H}$, et al. Action needed to improve equity and diversity in global eye health leadership. Eye. 2020;34:1051-4.

2. Plant GT, Mtanda AT, Arden GB, Johnson GJ. An epidemic of optic neuropathy in Tanzania: Characterization of the visual disorder and associated peripheral neuropathy. Journal of the Neurological Sciences. 1997;145(2):127-40.

3. Chaglasian M, Fingeret M, Gunvant Davey P, Huang W, Leung D, Ng E, et al. The development of a reference database with the Topcon 3D OCT-1 Maestro. Clinical Ophthalmology. 2018;12:849-57.

4. Girkin CA, Sample PA, Liebmann JM, Jain S, Bowd C, Becerra LM, et al. African Descent and Glaucoma Evaluation Study (ADAGES). Archives of Ophthalmology. 2010;128(5):541-50.

5. Poon LY-C, Antar H, Tsikata E, Guo R, Papadogeorgou G, Freeman M, et al. Effects of Age, Race, and Ethnicity on the Optic Nerve and Peripapillary Region Using Spectral-Domain OCT 3D Volume Scans. Translational vision science \& technology. 2018;7(6):12. 